PROCEEDINGS OF THE

AMERICAN MATHEMATICAL SOCIETY

Volume 127, Number 9, Pages 2561-2568

S 0002-9939(99)05291-0

Article electronically published on May 4, 1999

\title{
GENERALIZED DEDEKIND SYMBOLS ASSOCIATED WITH THE EISENSTEIN SERIES
}

\author{
SHINJI FUKUHARA
}

(Communicated by Dennis A. Hejhal)

\begin{abstract}
We have shown recently that the space of modular forms, the space of generalized Dedekind sums, and the space of period polynomials are all isomorphic. In this paper, we will prove, under these isomorphisms, that the Eisenstein series correspond to the Apostol generalized Dedekind sums, and that the period polynomials are expressed in terms of Bernoulli numbers. This gives us a new more natural proof of the reciprocity law for the Apostol generalized Dedekind sums. Our proof yields as a by-product new polylogarithm identities.
\end{abstract}

\section{INTRODUCTION}

In the previous paper [2] we have shown that the space $M_{2 k}$ of modular forms of weight $2 k$ on $S L(2, \mathbb{Z})$ is naturally and bijectively mapped to the space of even (resp. odd) Dedekind symbols which have polynomial (resp. Laurent polynomial) reciprocity law of degree $2 k-2$. We have also shown that the polynomials which appear in the reciprocity law coincide with period polynomials of the modular forms. We may illustrate the situation as follows:

the space of modular forms of weight $2 k$

$\downarrow \cong$

the space of even (resp. odd) generalized Dedekind symbols with

polynomial (resp. Laurent polynomial) reciprocity law of degree $2 k-2$

$\downarrow \cong$

the space of odd (resp. even) period

polynomials (resp. Laurent polynomials) of degree $2 k-2$.

Since the Eisenstein series are the most typical modular forms, it should be interesting to know which generalized Dedekind symbols might be assigned to them in the correspondences described above. In this note we will address this question and

Received by the editors October 7, 1997

1991 Mathematics Subject Classification. Primary 11F20, 11F67; Secondary 11F11, 11M35, $33 \mathrm{E} 20$.

Key words and phrases. Dedekind sum, Eisenstein series, polylogarithm.

The author wishes to thank Professor N. Yui for her useful advice.

(C)1999 American Mathematical Society 
give a complete answer to it. We will show that the Eisenstein series of weight $2 k$ correspond to the $(2 k-1)$-th Apostol generalized Dedekind sums [1]. Furthermore, we will prove that Laurent polynomials in the reciprocity law are related to the Hirzebruch $L$-polynomials (refer to Zagier [6, p. 158]), which are familiar objects to topologists. These correspondences may be schematically expressed as follows:

$$
\begin{gathered}
\text { the Eisenstein series of weight } 2 k \\
\downarrow \\
\text { the }(2 k-1) \text {-th Apostol generalized Dedekind sum } \\
\downarrow \\
-\frac{(2 k-2) !}{2 p q} \sum_{\substack{n=0 \\
n \text { even }}}^{2 k} \frac{B_{n} B_{2 k-n}}{n !(2 k-n) !} p^{n} q^{2 k-n}-\frac{B_{2 k}}{4 k} p^{-1} q^{-1} \\
\hline
\end{gathered}
$$

where and hereafter $B_{n}$ denotes the $n$-th Bernoulli number. This provides us with a natural alternative proof of the Apostol theorem [1, Theorem 1] on the reciprocity law of his generalized Dedekind sums (Theorem 6.4). This also presents another example of relationships between modular forms and topology of manifolds mentioned in Hirzebruch-Berger-Jung [4] (see also [3] and [5]).

In the course of the proof of the above correspondences, we also obtain new polylogarithm identities (Theorem 5.2). The polylogarithm function $L i_{k}(z)$ of order $k$ is defined by $L i_{k}(z)=\sum_{n=1}^{\infty} z^{n} / n^{k}(k \geq 1)$ for $|z|<1$, and then continued analytically onto $\mathbb{C}-[1, \infty)$. Note that $L i_{k}(1)=\zeta(k)$ with the Riemann zetafunction $\zeta(s)$. Our identities are

$$
\begin{aligned}
p^{2 k-3} \sum_{n=1}^{p-1} n L i_{2 k-1}\left(e^{2 \pi i n q / p}\right)-q^{2 k-3} \sum_{n=1}^{q-1} n L i_{2 k-1}\left(e^{-2 \pi i n p / q}\right) \\
=-\sum_{\substack{n=-1 \\
n \text { odd }}}^{2 k-1} \frac{(2 \pi i)^{2 k-1} B_{n+1} B_{2 k-n-1}}{2(n+1) !(2 k-n-1) !} p^{n} q^{2 k-2-n} \\
\quad-\frac{(2 \pi i)^{2 k-1} B_{2 k}}{4 k(2 k-2) !} p^{-1} q^{-1}+\frac{\zeta(2 k-1)}{2}\left(q^{2 k-2}-p^{2 k-2}\right)
\end{aligned}
$$

for positive integers $p, q$ such that $(p, q)=1$.

\section{Dedekind symbols ASSOciated With Modular Forms}

Throughout the paper, let $k$ denote a positive integer. Let $M_{2 k}$ denote the space of modular forms of weight $2 k$. For $f \in M_{2 k}$, let $f(\tau)=\sum_{n=0}^{\infty} a_{f}(n) e^{2 \pi i n \tau}$ be its Fourier expansion. The generalized Dedekind symbol $D_{f}$ associated to $f$ was first defined in [2, Definition 7.1]. Here we will give another expression for $D_{f}$. Let $p, q$ be integers such that $p \geq 1$ and $(p, q)=1$. We introduce a function $A_{f}$ :

$$
A_{f}(s ; p, q):=e^{\pi i s / 2} p^{s-1} \int_{0}^{\infty}\left\{f(i t+q / p)-a_{f}(0)\right\} t^{s-1} d t .
$$

It is easy to see that $A_{f}(s ; p, q)$ is well defined for $\Re(s) \gg 0$ and has a meromorphic 
continuation, say $A_{f}^{*}(s ; p, q)$, to all $s \in \mathbb{C}$. More explicitly, we have

$$
\begin{aligned}
A_{f}^{*}(s ; p, q)= & e^{\pi i s / 2} p^{s-1} \int_{t_{0}}^{\infty}\left\{f(i t+q / p)-a_{f}(0)\right\} t^{s-1} d t \\
& +e^{\pi i s / 2} p^{s-1} \int_{0}^{t_{0}}\left\{f(i t+q / p)-\frac{a_{f}(0)}{(p i t)^{2 k}}\right\} t^{s-1} d t \\
& -e^{\pi i s / 2} p^{s-1} a_{f}(0)\left\{\frac{t_{0}^{s}}{s}+\frac{t_{0}^{s-2 k}}{(p i)^{2 k}(s-2 k)}\right\}
\end{aligned}
$$

( $t_{0}>0$ arbitrary). The right-hand side of (2.1) is independent of the choice of $t_{0}$. It is plain that, using $(2.1), D_{f}(p, q)$ can be rewritten as: $D_{f}(p, q)=A_{f}^{*}(2 k-1 ; p, q)$.

\section{Dedekind symbols associated with the Eisenstein Series}

In this section we will obtain an explicit form for $D_{f}(p, q)$ when $f$ is the Eisenstein series of weight $2 k$. Let $G_{2 k}$ denote the Eisenstein series of weight $2 k$. It is well known that $G_{2 k}$ has the expansion:

$$
G_{2 k}(z)=-B_{2 k} /(4 k)+\sum_{m=1}^{\infty} \sum_{n=1}^{\infty} n^{2 k-1} e^{2 \pi i m n z} .
$$

The result is formulated as follows.

\section{Proposition 3.1.}

$$
D_{G_{2 k}}(p, q)=\frac{p^{2 k-2}(2 k-2) !}{(2 \pi i)^{2 k-1}}\left\{\sum_{l=1}^{p-1} \frac{l}{p} L i_{2 k-1}\left(e^{2 \pi i l q / p}\right)+\left(1-\frac{1}{2 p^{2 k-2}}\right) \zeta(2 k-1)\right\} .
$$

Proof. First we calculate $A_{G_{2 k}}(s ; p, q)$.

$$
\begin{aligned}
A_{G_{2 k}}(s ; p, q) & =e^{\pi i s / 2} p^{s-1} \int_{0}^{\infty}\left\{G_{2 k}(i t+q / p)+B_{2 k} /(4 k)\right\} t^{s-1} d t \\
& =e^{\pi i s / 2} p^{s-1} \int_{0}^{\infty}\left\{\sum_{m=1}^{\infty} \sum_{n=1}^{\infty} n^{2 k-1} e^{2 \pi i m n(i t+q / p)}\right\} t^{s-1} d t \\
& =e^{\pi i s / 2} p^{s-1} \int_{0}^{\infty} \sum_{m=1}^{\infty} \sum_{n=1}^{\infty} n^{2 k-1} e^{2 \pi i m n q / p} \int_{0}^{\infty} e^{-2 \pi m n t} t^{s-1} d t \\
& =e^{\pi i s / 2} p^{s-1} \frac{\Gamma(s)}{(2 \pi)^{s}} \sum_{m=1}^{\infty} \sum_{n=1}^{\infty} \frac{n^{2 k-1-s}}{m^{s}} e^{2 \pi i m n q / p} \\
& =e^{\pi i s / 2} p^{s-1} \frac{\Gamma(s)}{(2 \pi)^{s}} \sum_{m=1}^{\infty} \sum_{l=1}^{p} \sum_{j=0}^{\infty}(p j+l)^{2 k-1-s} \frac{1}{m^{s}} e^{2 \pi i m l q / p} \\
& =e^{\pi i s / 2} p^{2 k-2} \frac{\Gamma(s)}{(2 \pi)^{s}} \sum_{m=1}^{\infty} \sum_{l=1}^{p} \sum_{j=0}^{\infty}(j+l / p)^{2 k-1-s} \frac{1}{m^{s}} e^{2 \pi i m l q / p} \\
& =e^{\pi i s / 2} p^{2 k-2} \frac{\Gamma(s)}{(2 \pi)^{s}} \sum_{m=1}^{\infty} \sum_{l=1}^{p} \zeta(s+1-2 k, l / p) \frac{1}{m^{s}} e^{2 \pi i m l q / p} \\
& =e^{\pi i s / 2} p^{2 k-2} \frac{\Gamma(s)}{(2 \pi)^{s}} \sum_{l=1}^{p} \zeta(s+1-2 k, l / p) \sum_{m=1}^{\infty} \frac{1}{m^{s}} e^{2 \pi i m l q / p}
\end{aligned}
$$

where $\zeta(s, \alpha)$ denotes the Hurwitz zeta function. 
Since $D_{G_{2 k}}(p, q)=A_{G_{2 k}}^{*}(2 k-1 ; p, q)$, taking $s=2 k-1$ in the equation above, we obtain

$$
D_{G_{2 k}}(p, q)=i^{2 k-1} p^{2 k-2} \frac{\Gamma(2 k-1)}{(2 \pi)^{2 k-1}} \sum_{l=1}^{p} \zeta(0, l / p) \sum_{m=1}^{\infty} \frac{1}{m^{2 k-1}} e^{2 \pi i m l q / p} .
$$

Now noting that $\zeta(0, l / p)=-B_{1}(l / p)=-(l / p-1 / 2)$ and

$$
\sum_{l=1}^{p} \sum_{m=1}^{\infty}\left(1 / m^{2 k-1}\right) e^{2 \pi i m l q / p}=\zeta(2 k-1) / p^{2 k-2},
$$

we have

$$
\begin{aligned}
D_{G_{2 k}}(p, q) & =-\frac{i^{2 k-1} p^{2 k-2}(2 k-2) !}{(2 \pi)^{2 k-1}}\left\{\sum_{l=1}^{p} \frac{l}{p} \sum_{m=1}^{\infty} \frac{1}{m^{2 k-1}} e^{2 \pi i m l q / p}-\frac{1}{2 p^{2 k-2}} \zeta(2 k-1)\right\} \\
& =\frac{p^{2 k-2}(2 k-2) !}{(2 \pi i)^{2 k-1}}\left\{\sum_{l=1}^{p-1} \frac{l}{p} L i_{2 k-1}\left(e^{2 \pi i l q / p}\right)+\left(1-\frac{1}{2 p^{2 k-2}}\right) \zeta(2 k-1)\right\} .
\end{aligned}
$$

This completes our proof.

\section{RECIPROCITY LAW FOR $D_{f}(p, q)$}

Let us recall the definition [2, Definition 7.2] of $R(f)(p, q)$ for $f \in M_{2 k}$. To ease notations, we simply write $R_{f}(p, q)$ for $R(f)(p, q)$ in what follows.

$$
\begin{aligned}
R_{f}(p, q)= & \int_{\tau_{0}}^{i \infty}\left\{f(\tau)-a_{f}(0)\right\}(p \tau-q)^{2 k-2} d \tau \\
& +\int_{0}^{\tau_{0}}\left\{f(\tau)-\frac{a_{f}(0)}{\tau^{2 k}}\right\}(p \tau-q)^{2 k-2} d \tau \\
& -a_{f}(0)\left\{\frac{1}{(2 k-1) p}\left(p \tau_{0}-q\right)^{2 k-1}-\frac{1}{(2 k-1) q}\left(p-q / \tau_{0}\right)^{2 k-1}\right\} \\
& +a_{f}(0) p^{-1} q^{-1} \quad\left(\tau_{0} \in H \text { arbitrary }\right) .
\end{aligned}
$$

It was shown in $[2$, Lemma 7.2$]$ that

$$
D_{f}(p, q)-D_{f}(q,-p)=R_{f}(p, q)
$$

for positive integers $p, q$ such that $(p, q)=1$.

For $f \in M_{2 k}$, let $L_{f}$ be defined by:

$$
L_{f}(s)=\int_{0}^{\infty}\left\{f(i t)-a_{f}(0)\right\} t^{s-1} d t .
$$

Then $L_{f}(s)$ is well defined for $\Re(s) \gg 0$ and has an analytic continuation, say $L_{f}^{*}(s)$, to all $s \in \mathbb{C}$. More explicitly, we have

$$
\begin{aligned}
L_{f}^{*}(s)= & \int_{t_{0}}^{\infty}\left\{f(i t)-a_{f}(0)\right\} t^{s-1} d t+\int_{0}^{t_{0}}\left\{f(i t)-\frac{a_{f}(0)}{(i t)^{2 k}}\right\} t^{s-1} d t \\
& -a_{f}(0)\left\{\frac{t_{0}^{s}}{s}+\frac{(-1)^{k} t_{0}^{s-2 k}}{s-2 k}\right\} \quad\left(t_{0}>0\right. \text { arbitrary). }
\end{aligned}
$$


Lemma 4.1.

$$
\begin{aligned}
R_{f}(p, q)= & \sum_{n=0}^{2 k-2} i^{1-n}\left(\begin{array}{c}
2 k-2 \\
n
\end{array}\right) L_{f}^{*}(n+1) p^{n} q^{2 k-2-n} \\
& +\frac{a_{f}(0)}{2 k-1}\left\{p^{-1} q^{2 k-1}+p^{2 k-1} q^{-1}\right\}+a_{f}(0) p^{-1} q^{-1} .
\end{aligned}
$$

Proof. Expand the term $(p \tau-q)^{k-2}$ in the right-hand side of (4.1). Then arrange the terms according to the degrees in $p$. This gives the formula we are after.

\section{Reciprocity LAW FOR $D_{G_{2 k}}(p, q)$}

Applying Lemma 4.1, we can calculate $R_{f}(p, q)$ for $f=G_{2 k}$.

\section{Lemma 5.1.}

$$
\begin{aligned}
R_{G_{2 k}}(p, q)= & -\sum_{\substack{n=-1 \\
n \text { odd }}}^{2 k-1} \frac{(2 k-2) ! B_{n+1} B_{2 k-n-1}}{2(n+1) !(2 k-n-1) !} p^{n} q^{2 k-2-n} \\
& -\frac{(2 k-2) ! \zeta(2 k-1)}{2(2 \pi i)^{2 k-1}}\left(q^{2 k-2}-p^{2 k-2}\right)-\frac{B_{2 k}}{4 k} p^{-1} q^{-1}
\end{aligned}
$$

Proof. (This is essentially due to Zagier [7].) We use the well known fact that $L_{G_{2 k}}^{*}(s)=(2 \pi)^{-s} \Gamma(s) \zeta(s) \zeta(s-2 k+1)$. It is also known that $\zeta(1-n)=-B_{n} / n$, $\zeta(n)=-(2 \pi i)^{n} B_{n} / 2 n !(n>0$ even $)$ and $\zeta(1-n)=0(n>1$ odd $)$. We then have

$$
\begin{aligned}
R_{G_{2 k}}(p, q)= & \sum_{n=0}^{2 k-2} i^{1-n}\left(\begin{array}{c}
2 k-2 \\
n
\end{array}\right) L_{G_{2 k}}^{*}(n+1) p^{n} q^{2 k-2-n} \\
& -\frac{B_{2 k}}{4 k(2 k-1)}\left\{p^{-1} q^{2 k-1}+p^{2 k-1} q^{-1}\right\}-\frac{B_{2 k}}{4 k} p^{-1} q^{-1} \\
= & \sum_{n=0}^{2 k-2} i^{1-n}\left(\begin{array}{c}
2 k-2 \\
n
\end{array}\right) \frac{\Gamma(n+1)}{(2 \pi)^{n+1}} \zeta(n+1) \zeta(n-2 k+2) p^{n} q^{2 k-2-n} \\
& -\frac{B_{2 k}}{4 k(2 k-1)}\left\{p^{-1} q^{2 k-1}+p^{2 k-1} q^{-1}\right\}-\frac{B_{2 k}}{4 k} p^{-1} q^{-1} \\
& \sum_{n=0}^{2 k-2} i^{1-n}\left(\begin{array}{c}
2 k-2 \\
n
\end{array}\right) \frac{n !}{(2 \pi)^{n+1}} \frac{-(2 \pi i)^{n+1} B_{n+1}}{2(n+1) !} \frac{-B_{2 k-1-n}}{2 k-1-n} p^{n} q^{2 k-2-n} \\
& n \text { odd } \\
& -\frac{(2 k-2) ! \zeta(2 k-1)}{2(2 \pi i)^{2 k-1}}\left(q^{2 k-2}-p^{2 k-2}\right) \\
& -\frac{B_{2 k}}{4 k(2 k-1)}\left\{p^{-1} q^{2 k-1}+p^{2 k-1} q^{-1}\right\} \\
& -\frac{B_{2 k}}{4 k} p^{-1} q^{-1} \\
= & -\sum_{n=-1}^{2 k-1} \frac{(2 k-2) ! B_{n+1} B_{2 k-n-1}}{2(n+1) !(2 k-n-1) !} p^{n} q^{2 k-2-n} \\
& -\frac{(2 k-2) ! \zeta(2 k-1)}{2(2 \pi i)^{2 k-1}}\left(q^{2 k-2}-p^{2 k-2}\right)-\frac{B_{2 k}}{4 k} p^{-1} q^{-1} . \\
& \text { odd }
\end{aligned}
$$


From (4.2) and Lemma 5.1 above, we can deduce a new polylogarithm identity.

Theorem 5.2. For positive integers $p, q$ such that $(p, q)=1$, the identity (1.1) holds.

Proof. We obtain the following equation from Proposition 3.1, Lemma 5.1 and the identity (4.2) for $G_{2 k}$ :

$$
\begin{aligned}
& \frac{p^{2 k-2}(2 k-2) !}{(2 \pi i)^{2 k-1}} \sum_{l=1}^{p-1} \frac{l}{p} L i_{2 k-1}\left(e^{2 \pi i l q / p}\right)-\frac{q^{2 k-2}(2 k-2) !}{(2 \pi i)^{2 k-1}} \sum_{l=1}^{q-1} \frac{l}{q} L i_{2 k-1}\left(e^{-2 \pi i l p / q}\right) \\
= & -\sum_{\substack{n=-1 \\
n \text { odd }}}^{2 k-1} \frac{(2 k-2) ! B_{n+1} B_{2 k-n-1}}{2(n+1) !(2 k-n-1) !} p^{n} q^{2 k-2-n}-\frac{B_{2 k}}{4 k} p^{-1} q^{-1} \\
& +\frac{(2 k-2) ! \zeta(2 k-1)}{2(2 \pi i)^{2 k-1}}\left(q^{2 k-2}-p^{2 k-2}\right) .
\end{aligned}
$$

The assertion of the theorem then follows from this.

Corollary 5.3. For a positive integer $p$, set $\xi=e^{2 \pi i / p}$. Then we have

$$
\begin{aligned}
\sum_{n=1}^{p-1} n L i_{2 k-1}\left(\xi^{n}\right)= & -\frac{(2 \pi i)^{2 k-1}}{2} \sum_{\substack{n=-1 \\
n \text { odd }}}^{2 k-1} \frac{B_{n+1} B_{2 k-n-1}}{(n+1) !(2 k-n-1) !} p^{1-n} \\
& -\frac{(2 \pi i)^{2 k-1} B_{2 k}}{4 k(2 k-2) !} p^{-2 k+2}+\frac{\zeta(2 k-1)}{2}\left(p^{-2 k+3}-p\right) .
\end{aligned}
$$

\section{Apostol generalized Dedekind sums}

Recall that any generalized Dedekind symbol $D(p, q)$ splits uniquely as $D(p, q)=$ $D^{+}(p, q)+D^{-}(p, q)$ so that $D^{+}(p,-q)=D^{+}(p, q)$ and $D^{-}(p,-q)=-D^{-}(p, q)$. We call $D^{+}$(resp. $D^{-}$) the even (resp. odd) part of $D$. In this section we will show that the odd part of $D_{G_{2 k}}(p, q)$ is essentially the Apostol generalized Dedekind sum and that Theorem 5.2 corresponding to the odd part is the Apostol reciprocity law.

Let $\bar{B}_{p}(x)$ denote the $p$-th Bernoulli function which is given by the Fourier expansion

$$
\bar{B}_{p}(x)=-p !(2 \pi i)^{-p} \sum_{\substack{m=-\infty \\ m \neq 0}}^{+\infty} m^{-p} e^{2 \pi i m x}
$$

For $0 \leq x<1, \bar{B}_{p}(x)$ reduces to the $p$-th Bernoulli polynomial. For integers $h, k$ such that $(h, k)=1$, Apostol [1] introduced the $p$-th generalized Dedekind sums $s_{p}(h, k)$ by

$$
s_{p}(h, k)=\sum_{\mu=1}^{k-1}(\mu / k) \bar{B}_{p}(h \mu / k)
$$

The sum $s_{p}(h, k)$ coincides with the original Dedekind sum when $p=1$. Apostol proved the reciprocity law for these generalized Dedekind sums:

Theorem 6.1. For odd $p,(h, k)=1$, we have the reciprocity law

$$
(p+1)\left\{h k^{p} s_{p}(h, k)+k h^{p} s_{p}(k, h)\right\}=\sum_{s=0}^{p+1}\left(\begin{array}{c}
p+1 \\
s
\end{array}\right)(-1)^{s} B_{s} h^{s} B_{p+1-s} k^{p+1-s}+p B_{p+1} .
$$


Now we will calculate the odd part $D_{G_{2 k}}^{-}(p, q)$. For this, we will use the odd part of the identity (4.2) ([2]):

$$
D_{f}^{-}(p, q)-D_{f}^{-}(q,-p)=R_{f}^{-}(p, q) .
$$

(Here again we simply write $R_{f}^{-}(p, q)$ for $R^{-}(f)(p, q)$.) Indeed, we will obtain an explicit form for $D_{G_{2 k}}^{-}(p, q)$ (Lemma 6.2) and $R_{G_{2 k}}^{-}(p, q)$ (Lemma 6.3), and finally the reciprocity law (Theorem 6.4 ).

Lemma 6.2. We have

$$
D_{G_{2 k}}^{-}(p, q)=-\frac{p^{2 k-2}}{2(2 k-1)} \sum_{l=1}^{p-1} \frac{l}{p} \bar{B}_{2 k-1}(q l / p) .
$$

Proof.

$$
\begin{aligned}
D_{G_{2 k}}^{-}(p, q) & =\frac{1}{2}\left\{D_{G_{2 k}}(p, q)-D_{G_{2 k}}(p,-q)\right\} \\
& =\frac{p^{2 k-2}(2 k-2) !}{2(2 \pi i)^{2 k-1}} \sum_{l=1}^{p} \frac{l}{p}\left\{\sum_{m=1}^{\infty} \frac{1}{m^{2 k-1}}\left(e^{2 \pi i m l q / p}-e^{-2 \pi i m l q / p}\right)\right\} \\
& =\frac{p^{2 k-2}(2 k-2) !}{2(2 \pi i)^{2 k-1}} \sum_{l=1}^{p-1} \frac{l}{p} \sum_{\substack{m=-\infty \\
m \neq 0}}^{+\infty} \frac{1}{m^{2 k-1}} e^{2 \pi i m l q / p} \\
& =\frac{p^{2 k-2}(2 k-2) !}{2(2 \pi i)^{2 k-1}} \sum_{l=1}^{p-1} \frac{l}{p}\left\{-\frac{(2 \pi i)^{2 k-1}}{(2 k-1) !} \bar{B}_{2 k-1}(l q / p)\right\} \\
& =-\frac{p^{2 k-2}}{2(2 k-1)} \sum_{l=1}^{p-1} \frac{l}{p} \bar{B}_{2 k-1}(l q / p) .
\end{aligned}
$$

Next we will calculate $R_{G_{2 k}}^{-}(p, q)$.

Lemma 6.3. We have

$$
R_{G_{2 k}}^{-}(p, q)=-\sum_{\substack{n=-1 \\ n \text { odd }}}^{2 k-1} \frac{(2 k-2) ! B_{n+1} B_{2 k-n-1}}{2(n+1) !(2 k-n-1) !} p^{n} q^{2 k-2-n}-\frac{B_{2 k}}{4 k} p^{-1} q^{-1} .
$$

Proof. Since $R_{G_{2 k}}^{-}(p, q)=\left\{R_{G_{2 k}}(p, q)-R_{G_{2 k}}(p,-q)\right\} / 2, R_{G_{2 k}}^{-}(p, q)$ is the odd part of $R_{G_{2 k}}(p, q)$. Hence the assertion follows from Lemma 5.1.

Now we apply the identity (6.1) to $G_{2 k}$, and the results in Lemmas 6.2 and 6.3 to obtain the following formula:

Theorem 6.4. For positive integers $p, q$ such that $(p, q)=1$,

$$
\begin{aligned}
p^{2 k-2} & \sum_{l=1}^{p-1} \frac{l}{p} \bar{B}_{2 k-1}(q l / p)-q^{2 k-2} \sum_{l=1}^{q-1} \frac{l}{q} \bar{B}_{2 k-1}(-p l / q) \\
= & \sum_{\substack{n=-1 \\
n \text { odd }}}^{2 k-1} \frac{(2 k-1) ! B_{n+1} B_{2 k-n-1}}{(n+1) !(2 k-n-1) !} p^{n} q^{2 k-2-n}+\frac{(2 k-1) B_{2 k}}{2 k} p^{-1} q^{-1} .
\end{aligned}
$$


Noting that $\bar{B}_{p}(-x)=-\bar{B}_{p}(x)$ for odd $p$, and that $B_{s}=0$ for odd $s>1$, we see easily that this theorem indeed coincides with the odd part of the Apostol Theorem 6.1 .

\section{REFERENCES}

[1] Apostol, T. M.: Generalized Dedekind sums and transformation formulae of certain Lambert series. Duke Math. J. 17, 147-157 (1950) MR 11:641g

[2] Fukuhara, S.: Modular forms, generalized Dedekind symbols and period polynomials. Math. Ann. 310, 83-101 (1998) CMP 98:07

[3] Hirzebruch, F.: The signature theorem: reminiscences and recreation. In: Prospects in Mathematics (Ann. Math. Studies No. 70), pp. 3-31. Princeton 1971 MR 51:4265

[4] Hirzebruch, F., Berger, T., Jung, R.: Manifolds and Modular forms. Bonn: Vieweg 1992 MR 94d:57001

[5] Hirzebruch, F., Zagier, D.: The Atiyah-Singer theorem and elementary number theory. Berkeley: Publish or Perish 1974 MR 58:31291

[6] Zagier, D.: Higher dimensional Dedekind sums. Math. Ann. 202, 149-172 (1973) MR 50:9801

[7] Zagier, D.: Periods of modular forms and Jacobi theta functions. Invent. Math. 104, 449-465 (1991) MR 92e:11052

Department of Mathematics, Tsuda College, Tsuda-machi 2-1-1, Kodaira-shi, Tokyo 187, JAPAN

E-mail address: fukuhara@tsuda.ac.jp 